\title{
Study on vertical dynamic vehicle-track interactions using the TRADYS test facility and computer simulation
}

\author{
M. Miwa ${ }^{1} \&$ A. Yoshimura ${ }^{2}$ \\ ${ }^{1}$ Technology Research and Development Department, \\ General Technology Division, Central Japan Railway Company, Japan \\ ${ }^{2}$ School of Computer Science, Tokyo University of Technology, Japan
}

\begin{abstract}
It is important when studying vehicle-track dynamic interactions to make clear the characteristics of many physical and/or structural parameters of a track. However, it is difficult to estimate them with accuracy only through measurements under a real train load on site. In this paper, we describe an approach using two tools of a test facility and computer simulation. The Track and Structural Dynamics Simulator (TRADYS) is a unique mechanical vibration exciter equipped with hydraulic actuators between a train and a track. The train has the same masses and suspensions as the latest Japanese high speed railway vehicle. We can control arbitrarily the vertical relative displacements between rails and wheels through the directions inputted to the actuator. These relative displacements are supposed to represent a railway track irregularity. In the experiments, dynamic wheel loads, displacements and accelerations of each part of a track are measured as the outputs. In addition to the experiments, computer simulation is carried out. A theoretical model for this special test facility including the actuator is constructed. In this paper the measured physical values and computed solutions are compared, and the accuracy of the parameter values of the track structure components is discussed.
\end{abstract}

Keywords: vehicle-track interaction, dynamic model, test facility, computer simulation, parameter estimation. 


\section{Introduction}

We have researched not only vehicle-track dynamic interactions but also various railway technological issues using three instruments and combining them effectively as shown in Fig. 1. In the disciplines of vehicle-track dynamics, characteristics of dynamic wheel load, ground vibration and effects of unsprungmass of a vehicle determined before with the high-speed experimental train " $300 X "$ in Miwa and Nakamura [1]. To enhance these researches aiming at more improvement of running stability and riding comfort, reduction of ground vibration and track maintenance, we introduced a unique test facility and developed a computer simulation program as described below.

The test facility in this paper generates dynamic load at the fixed point on a track while the real train works as a moving load. In the former, we can change load arbitrarily to estimate track parameters conveniently. The parameters are estimated with the computer simulation which simulates vehicle/track behavior under dynamic load at the fixed point. Then moving load is simulated with the estimated parameters and its accuracy is verified with field data.

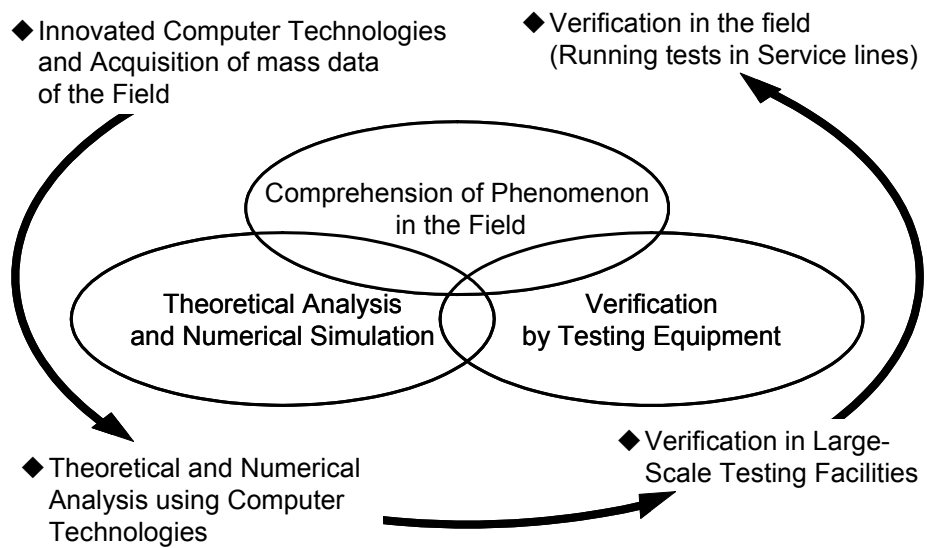

Figure 1: $\quad$ Basic cycle for R\&D of railways.

\section{Track and Structural Dynamics Simulator, TRADYS}

The full view of the test facility, Track and Structural Dynamics Simulator (TRADYS), is shown in Fig. 2. Its total weight and bogies are the same as the real vehicle, the Series 700, of the Tokaido Shinkansen. The leftmost axle in Fig. 2 is replaced with the vibration exciter, and it is called as the Dynamics Testing Bogie. Its detailed view is shown in Fig. 3. A pair of hydraulic actuators and dead-weight which are equivalent to the unsprung-mass of the vehicle are set here substituting for the original axle. The unsprung-mass is changeable from 1.4 to 2.3 ton while its value of the Series 700 is approximately 1.8 ton. Actuators are mounted on rails through jigs of the wheel tread shape, and they give relative 
displacement between rails and wheels (unsprung-mass) forcibly at the fixed point. The displacement given by the actuators represents track irregularities, and therefore, the excitation force is generated as a result of vehicle-track interaction based on cause-and-effect relationship (see Fig. 4). The excitation force is considered to equivalently correspond to the one due to a moving vehicle on a track irregularity. In addition, displacement is given with regular waves, such as sine-waves, and random waves. Therefore it is easy to estimate parameters of track components by changing input waves arbitrarily.

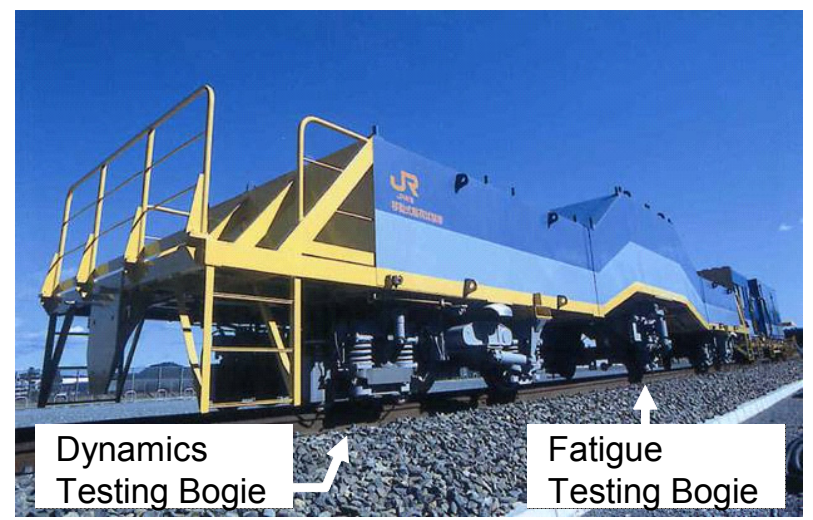

Figure 2: $\quad$ Track and Structural Dynamics Simulator, TRADYS test facility.

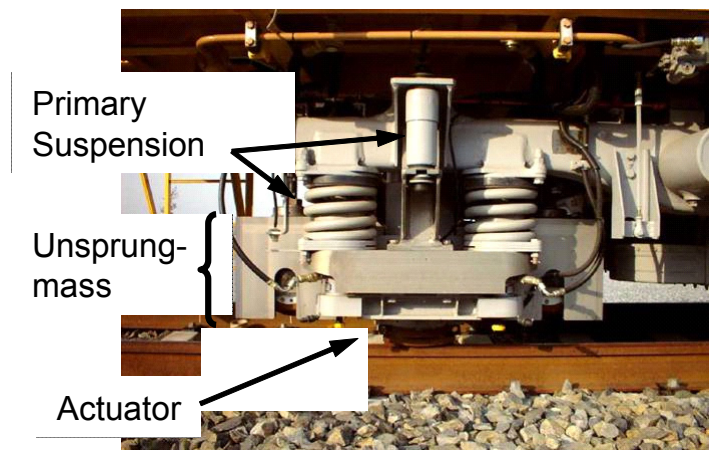

Figure 3: Dynamics testing bogie of TRADYS.

\section{Mathematical model for the TRADYS test facility}

Figure 4 shows the mathematical simulation model of the TRADYS test facility. The vehicle model consists of a rigid carbody, a rigid bogie and a rigid wheel. They have the same masses and suspensions as the latest Japanese high speed railway vehicle. The track consists of a single rail supported discretely by sleepers. The sleeper is modeled as a lumped mass. The pad spring and ballast spring are considered to be one-dimensional elements. The railpad works as a 
viscoelastic foundation with linear elastic stiffness and viscous damping. The ballast is assumed to consist of several layers (usually three layers) having each different effective mass. They are also connected by linear springs and viscous dampers in parallel. The roadbed is fixed to a ground.

In the following, a brief description of the equations of motions of the above dynamic models of a vehicle and a track with the constraint on the motion due to the forced relative displacement is given.

The equations of motion of the vehicle model can be written as

$$
\boldsymbol{M}_{V} \ddot{\boldsymbol{u}}_{V}(t)+\boldsymbol{C}_{V} \dot{\boldsymbol{u}}_{V}(t)+\boldsymbol{K}_{V} \boldsymbol{u}_{V}(t)=-P_{d y n}(t)\left(\begin{array}{l}
0 \\
0 \\
1
\end{array}\right)
$$

where $\boldsymbol{u}_{V}(t)$ is the displacement vector of the vehicle model (Yoshimura [2]). $\boldsymbol{M}_{V}, \boldsymbol{C}_{V}, \boldsymbol{K}_{V}$ are the mass, damping and stiffness matrices of a vehicle, respectively. $P_{d y n}(t)$ is an external dynamic force acting between a rail and a wheel due to an excitation by the actuator.

We focus on the vibration with predominant components in the frequency range from $0 \mathrm{~Hz}$ to $100 \mathrm{~Hz}$ caused by the vehicles running on tracks on the ground surface, so the rail is modeled as a simple Bernoulli-Euler beam. In the finite element analysis the rail beam is divided into $\mathrm{N}$ elements $(\mathrm{N}+1$ nodes), as shown in Figure 4. The length of each element is equally $h$. The Bernoulli-Euler beam element is a two-node element with two degrees of freedom for each node. The nodal coordinates of the element is described as the vector form:

$$
\boldsymbol{u}_{i}=\left(u_{i}, h \theta_{i}, u_{i+1}, h \theta_{i+1}\right)^{T} \quad i=0,1, \cdots, N-1
$$

where $u$ and $\theta$ are the vertical displacement and the rotational angle respectively, $i$ and $i+1$ are the global node numbers at each side of the $i$ th element.

The equations of motion of the track model are obtained as follows.

$$
\boldsymbol{M}_{R} \ddot{\boldsymbol{u}}_{R}(t)+\boldsymbol{C}_{R} \dot{\boldsymbol{u}}_{R}(t)+\boldsymbol{K}_{R} \boldsymbol{u}_{R}(t)=P_{d y n}(t)\left(\begin{array}{l}
\boldsymbol{a} \\
0
\end{array}\right)
$$

where $\boldsymbol{M}_{R}, \boldsymbol{C}_{R}, \boldsymbol{K}_{R}$ are the mass, damping and stiffness matrices of the track model respectively. $\boldsymbol{a}$ is a vector corresponding to $x_{w}$ which represents the position of a wheel on a rail and defined as follows, when a wheel is on the $i$ th finite element.

$$
\begin{aligned}
& \begin{array}{llllllllll}
1 & \cdots & 2 i-2 & 2 i-1 & 2 i & 2 i+1 & 2 i+2 & 2 i+3 & \cdots & 2(N-1)
\end{array} \\
& \boldsymbol{a}=\left(0, \cdots, \quad 0, N_{1}(\xi), N_{2}(\xi), N_{3}(\xi), N_{4}(\xi), \quad 0, \cdots, \quad 0\right)^{T}
\end{aligned}
$$


Using the vector $\boldsymbol{a}, u\left(x_{w}, t\right)$ the vertical displacement of the rail at the exciting position can be expressed as

$$
u\left(x_{w}, t\right)=\boldsymbol{a}^{T} \boldsymbol{u}_{0}^{*} \quad \text { where } \quad \boldsymbol{u}_{0}^{*}=\left(u_{1}, h \theta_{1}, \cdots, u_{N-1}, h \theta_{N-1}\right)^{T}
$$

Then the constraint on the vehicle-track motion due to the forced relative displacement can be written as the following algebraic equation.

$$
u\left(x_{w}, t\right)+r(t)=y_{w}(t)
$$

where $y_{w}(t)$ is the vertical displacement of a wheel at the position of excitation. $r(t)$ is the forced relative displacement by actuator.

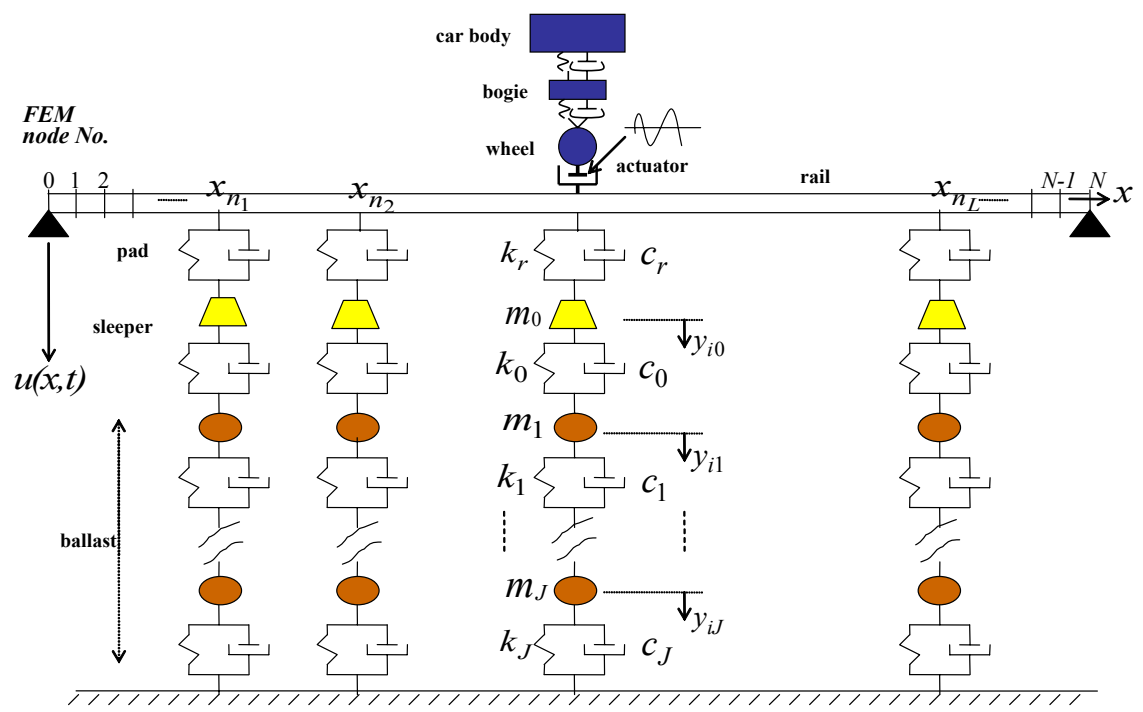

Figure 4: $\quad$ Mathematical simulation model of TRADYS test facility.

\section{Numerical methods for solution}

As described above, we have obtained the system represented by the two differential eqns (1), (3) and one algebraic constraint eqn (5) for the mathematical simulation model of the TRADYS test facility. This is a differential-algebraic equation, DAE. However, it is well known that it is sometimes difficult to solve the DAE directly and to obtain a numerical stable solution. From the point of view of the numerical solution, it is desirable for the DAE to have a differential index which is as small as possible. So we have tried to reduce the index. 
By differentiating the constraint eqn (5), we get

$$
\dot{u}\left(x_{w}, t\right)+\dot{r}(t)=\dot{y}_{w}(t)
$$

However, one of the difficulties with reducing the index in this way is that the numerical solution of the resulting system needed no longer satisfy the original constraints exactly. So in practice, we have adopted the following differential equation obtained by combining eqns (5) and (6).

$$
2 \alpha\left(\dot{y}_{w}(t)-\dot{u}\left(x_{w}, t\right)-\dot{r}(t)\right)+\beta^{2}\left(y_{w}(t)-u\left(x_{w}, t\right)-r(t)\right)=0
$$

where $\alpha, \beta$ are adjustable constants and their optimum values are to be determined by trial and error, observing the numerical results of the simulation.

As a result, it is required for us to solve the simultaneous differential eqns (1), (3) and (7). In this study we applied the well-known Newmark- $\beta$ method, one of the direct integration methods, to solve the problem. The above constants $\alpha, \beta$ were taken to $\alpha=1.0, \beta=1.0$ as optimum values by which numerical stability is attained successfully.

\section{Examples of experiments in the TRADYS}

The TRADYS and test track are installed in the Komaki R\&D Complex (Masuda [4]) of Central Japan Railway Company. All kinds of concrete sleepers which are used in the Tokaido Shinkansen Line are laid in the test track. Test results of the most standard track of the Tokaido Shinkansen shown in Table 1 are described as an example.

Table 1: $\quad$ Major specifications of tested track.

\begin{tabular}{|l|l|}
\hline \multicolumn{1}{|c|}{ item } & \multicolumn{1}{c|}{ specification } \\
\hline Rail & JIS $60 \mathrm{~kg}$ \\
\hline rail pad & $50 \mathrm{MN} / \mathrm{m}(\mathrm{t}=12 \mathrm{~mm})$ \\
\hline sleeper spacing & $581 \mathrm{~mm}$ \\
\hline ballast depth & $30 \mathrm{~cm}$ (below sleeper) \\
\hline
\end{tabular}

Track and rail surface irregularities which are measured by inspection cars are inputted as the forced displacement for hydraulic actuators. The included wavelength is ranges form $0.5 \mathrm{~m}$ to $150 \mathrm{~m}$. The assuming train speed is $270 \mathrm{~km} / \mathrm{h}$, and the unsprung-mass of the TRADYS weighs 1.8ton.

Experimental data are shown in Fig. 5. Discussions about them are described in comparison to numerical simulation results in the next section.

\section{Results of numerical simulations}

Results of numerical simulations are shown in Fig. 6. The forced displacement of the numerical simulation (Fig. 6(a)) is calculated as an output data. It matches very well with the input data shown in Fig. 5(a). It means that the numerical stable solution is obtained here as described in the Section 4. 


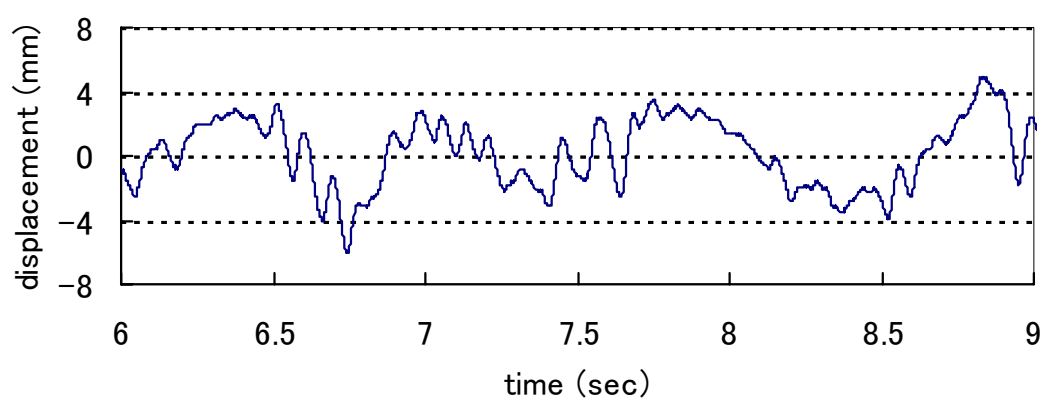

(a) forced displacement by actuator

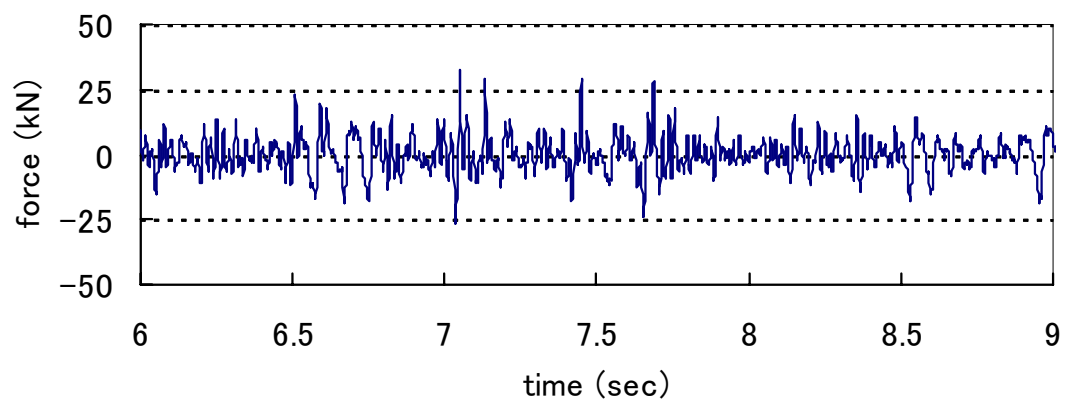

(b) excitation force by actuator

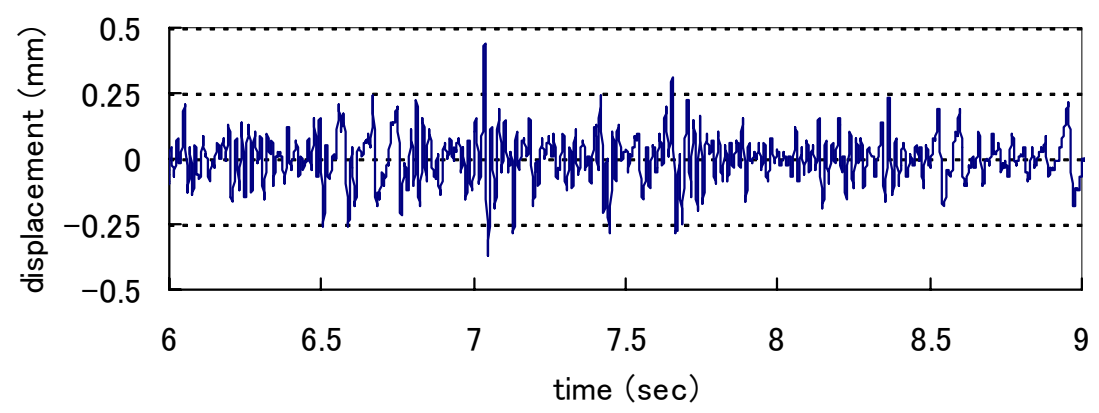

(c) displacement of rail

Figure 5: Experimental data in the TRADYS.

In comparison with the input data shown in Fig. 6(a) which corresponds to the track irregularity, higher frequency waves become dominant in the output data shown in Fig. 6(b) which corresponds to the dynamic wheel load. This can be similarly described in the case of TRADYS, and then Figs. 5(b) and 6(b) coincide with each other relatively well. Similar coincidence is observed for Figs. 5(c) and 6(c) which correspond to rail displacement.

The Spectral densities of TRADYS and simulation are shown in Fig. 7. The forced displacement by the actuator of each case matches with each other 
significantly (Fig. 7(a)). The spectrums lower than $20 \mathrm{~Hz}$ are resulted by track vertical irregularities which are caused by unequal settlement of sleepers, whereas those of higher than $20 \mathrm{~Hz}$ are resulted by very small unevenness of rail surface which are stamped unavoidably by the production process (Miwa and Nakamura [1]). The dip near around $20 \mathrm{~Hz}$ is resulted by measuring amplitude characteristics of the track inspection vehicles of two kinds, therefore it is not an essentially significant phenomenon.

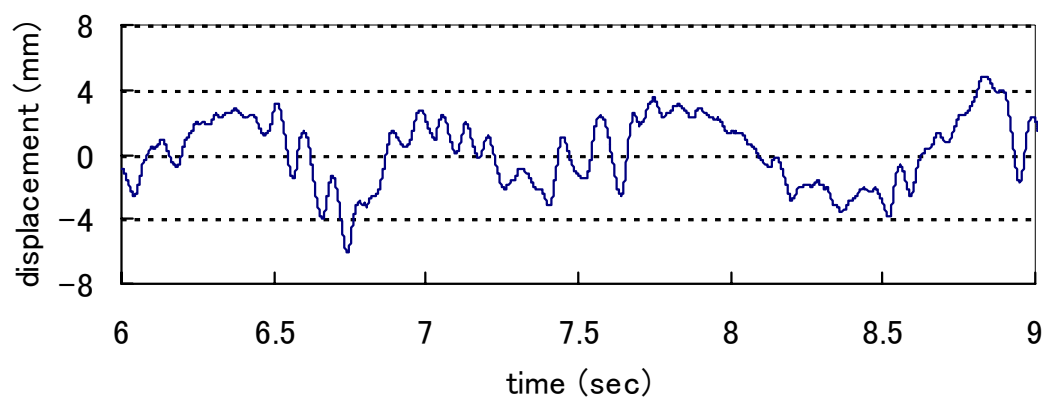

(a) forced displacement by actuator

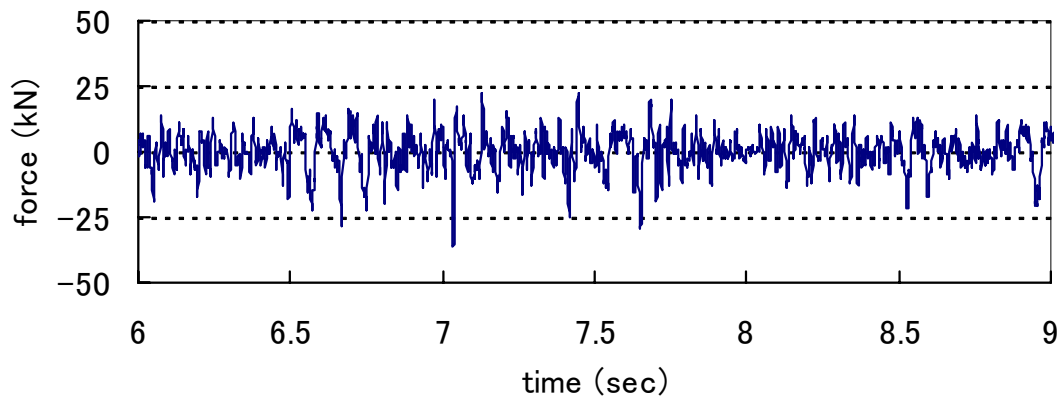

(b) excitation force by actuator

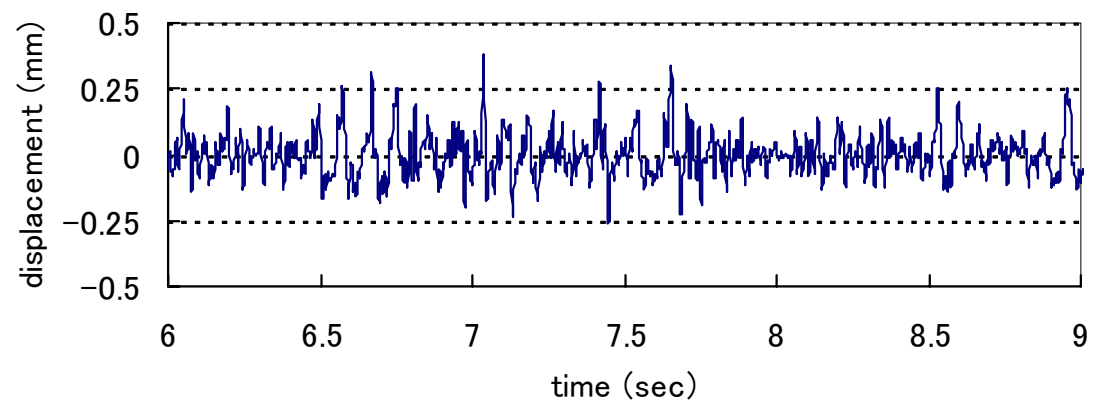

(c) displacement of rail

Figure 6: Numerical simulation data. 


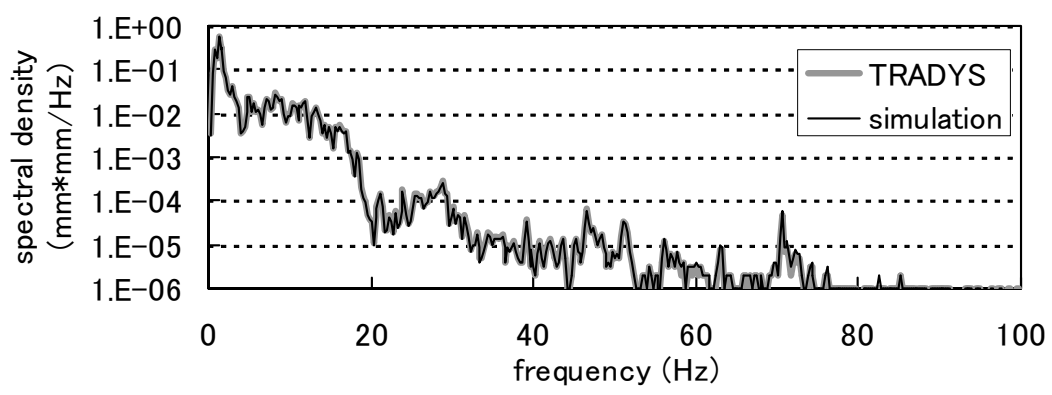

(a) forced displacement by actuator
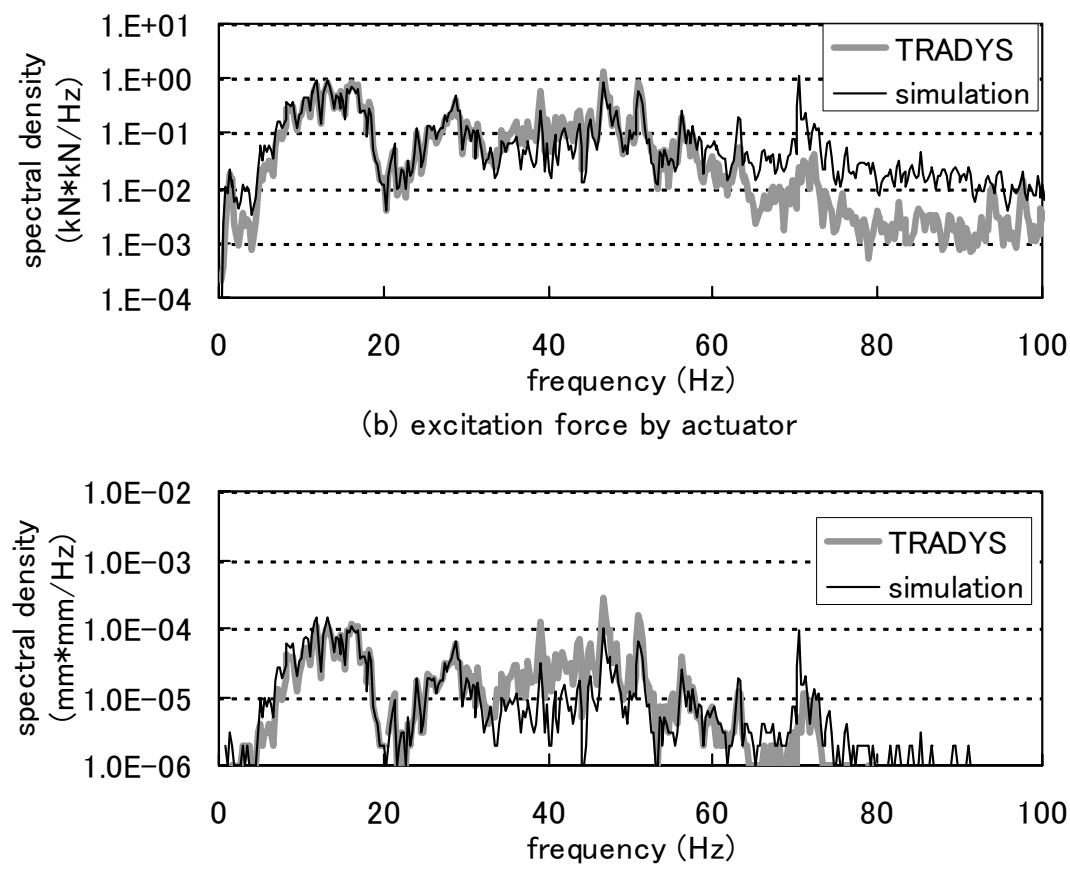

(c) displacement of rail

Figure 7: $\quad$ Power spectra of the TRADYS and simulation data.

It is observed that the excitation force by the actuator in Fig. 7(b) is amplified mainly from $50 \mathrm{~Hz}$ to $60 \mathrm{~Hz}$ based on the input shown in Fig. 7(a). The reason is that this phenomenon is affected by the transfer function (Miwa [1]) of mass-spring system which consists of vehicle unsprung-mass and track stiffness. The track in the Tokaido Shinkansen Line has appropriate damping factor. Therefore the transfer function forms a gently sloping convex, not a steep peak called as resonance. Some peaks at $47 \mathrm{~Hz}, 51 \mathrm{~Hz}, 56 \mathrm{~Hz}$, etc, depend on the characteristics of the input data in Fig. 7(a) which has these peaks at the same frequencies in origin. In comparison between TRADYS and simulation in Fig. 
7(b), they matches well in the frequency range of lower than $30 \mathrm{~Hz}$. Slight difference between them is seen in the frequency range between $30 \mathrm{~Hz}$ and $50 \mathrm{~Hz}$, and significant deviations occur in higher than $60 \mathrm{~Hz}$. Referring to accuracy, higher than $60 \mathrm{~Hz}$, the simulation is considered to be more faithful than the TRADYS judging by the field test result (Miwa [1]). The reason why the TRADYS value is lower is that the response of load sensor comes short in higher frequency. So we are planning an improvement in the experimental method.

In the Rail displacement in Fig. 7(c), they match well with each other in lower than $30 \mathrm{~Hz}$ similar with the case in Fig. 7(b). The difference which is seen in higher than $60 \mathrm{~Hz}$ is smaller than that of the actuator force.

\section{Conclusions}

It has become possible for us to estimate complicated parameters in track dynamic model with efficiently combining two tools; (1) the TRADYS test facility which has a special vibration exciter with the same mass-spring systems as the real high-speed railway vehicle, and (2) the computer simulation in which the TRADYS on track is modeled. Moreover, adding field tests with real vehicle and track to them, we can establish powerful approaches to solve the vertical vehicle-track dynamic interaction problem.

We are now planning to extend the simulation including soil foundation model. Special roadbed for these experiments has been equipped in our research complex.

\section{References}

[1] Miwa, M. \& Nakamura, S., High speed running test and the new testing equipment for wheel/rail dynamic interaction, Proc. of Int. Symposium on Speed-up and Service Technology for Railway and Maglev Systems (STECH'03), JSME, pp.489-493, 2003.

[2] Yoshimura, A. \& Ishida, M., A study on parameter estimation methods in the railway vehicle and track dynamic model, Computers in Railways VIII (COMPRAIL2002), pp.201-210, 2002.

[3] Bathe, K.J., Finite Element Procedures, Prentice-Hall, Inc., 1996.

[4] Masuda, Y., Technology development at JR Central. International Schienen der Welt, May 2003, pp.10-17, 2003. 